\title{
A rare cardiac finding in a morbidly obese patient with severe hypertension
}

\author{
Anna Maria Vittoria Fiore · Giorgia Michela Marinoni • \\ Alessandro Piccione • Maria Adelaide Marini • \\ Monica D'Adamo · Renato Lauro • Paolo Sbraccia
}

Received: 30 August 2010/Accepted: 23 September 2010/Published online: 9 October 2010

(c) SIMI 2010

\section{Case presentation}

Dr. Fiore, Dr. Marinoni: A 46-year-old morbidly obese man was admitted to our emergency department (ED) with the acute onset of epigastric pain, nausea, and vomiting. He reported having a 10-year history of hypertension treated with doxazosin, atenolol, ramipril, and potassium canrenoate; in addition, during the last 2 years, he was treated with overnight continuous positive airway pressure (C-PAP) device for obstructive sleep apnea syndrome. He had never smoked or used illicit drugs.

On examination, he appeared anxious. The height was $190 \mathrm{~cm}$, the weight $155 \mathrm{~kg}$, and the body-mass index (BMI, the weight in kilograms divided by the square of the height in meters) 42.9. The abdomen was soft and obese, with mild-to-moderate tenderness in the epigastrium, with no rebound tenderness or guarding. The bowel sounds were normal. The lungs were clear to auscultation, and the first and second heart sounds were faint but normal; a diastolic murmur (grade 1/6) was heard at the right sternal border. The temperature was $36.1^{\circ} \mathrm{C}$, heart rate 97 beats per minute, blood pressure was $210 / 115 \mathrm{mmHg}$, respiratory rate 20 breaths per minute and oxygen saturation $98 \%$ while he was breathing ambient air. The sclerae were anicteric.

Routine blood measurements revealed a hypochromic microcytic anemia (hemoglobin: $9.6 \mathrm{~g} / \mathrm{dl}$; hematocrit: $30 \%$; mean corpuscular volume: $75 \mathrm{fl}$ ) and increased

A. M. V. Fiore and G. M. Marinoni contributed equally to this work.

A. M. V. Fiore - G. M. Marinoni - A. Piccione ·

M. A. Marini · M. D’Adamo · R. Lauro · P. Sbraccia ( $ه)$

Department of Internal Medicine,

University of Rome "Tor Vergata",

Via Montpellier, 1, 00133 Rome, Italy

e-mail: sbraccia@med.uniroma2.it values of the classical markers of inflammation (erythrocyte sedimentation rate: $99 \mathrm{~mm} / \mathrm{h}$; C-reactive protein: $13.1 \mathrm{mg} / \mathrm{l})$. A complete blood count, serum levels of glucose, creatinine, total protein, albumin, globulin, troponin I, creatine kinase and creatine kinase isoenzymes, lactate dehydrogenase, amylase, lipase and results of liver-function tests, and serum protein electrophoresis were normal. An electrocardiogram was normal. The patient was treated with intravenous labetalol and proton-pump inhibitors.

Although symptoms, physical examination, and anemia were consistent with peptic ulcer or erosive gastritis, an esophagogastroduodenoscopy was unremarkable.

The initial symptoms improved gradually during the following $24 \mathrm{~h}$, although mild epigastric discomfort persisted. However, several hypertensive crises (systolic and diastolic values in a range of 180-220 and 110-140 $\mathrm{mmHg}$, respectively) occurred that required labetalol infusion for 3 days. Upon labetalol withdrawal, therapy with doxazosin (16 mg daily), transdermal clonidine (10 mg weekly), atenolol (200 mg daily), spironolactone (50 mg daily), furosemide (50 mg daily), amlodipine (10 mg daily), ramipril (10 mg daily) was gradually set, still with poor blood pressure control.

\section{Preliminary diagnosis}

Dr. Marini, Dr. Piccione: Although the patient was morbidly obese and had sleep apnea, two well-known conditions causing hypertension, stage 2 [1] hypertensive crises resistant to treatment prompted us to screen for other identifiable causes of hypertension, with the inevitable strong caveat of drug interference. Screening for primary aldosteronism was negative (plasma aldosterone-to-renin ratio of 16 ; normal value $<25$ ). Furthermore, in order to 
Table 1 24-h urinary catecholamines and their metabolites

\begin{tabular}{lcc}
\hline & $\begin{array}{l}\text { Patient's } \\
\text { values }\end{array}$ & $\begin{array}{l}\text { Normal } \\
\text { values }\end{array}$ \\
\hline Normetanephrine $(\mu \mathrm{g})$ & $1,563.0$ & $0-390$ \\
Metanephrine $(\mu \mathrm{g})$ & 404.0 & $0-320$ \\
Vanillylmandelic acid $(\mathrm{mg})$ & 9.2 & $1.8-6.7$ \\
Homovanillic acid $(\mathrm{mg})$ & 5.0 & $2-7.4$ \\
Epinephrine $(\mu \mathrm{g})$ & 22.1 & $0-20$ \\
Norepinephrine $(\mu \mathrm{g})$ & 228.8 & $23-105$ \\
Dopamine $(\mu \mathrm{g})$ & 369.2 & $190-450$ \\
\hline
\end{tabular}

rule out the presence of a pheochromocytoma, 24-h urinary catecholamines concentrations were determined (Table 1). Based on the values above the upper normal limits, a contrast-enhanced CT scan of the abdomen was performed with overall negative results and no adrenal masses. However, during the procedure a significant pericardial effusion was noted (Fig. 1a).

\section{Imaging and pathological diagnosis}

Dr. D'Adamo, Dr. Sbraccia: To better define the pericardial effusion characteristics, a transthoracic echocardiocardiogram was performed that unexpectedly showed a heterogeneous mass $(5.5 \mathrm{~cm}$ by $3.5 \mathrm{~cm})$ occupying the right atrium and intermittently occluding the tricuspid ostium (Fig. 1b). A subsequent cardiac CT showed a $51.5 \mathrm{~mm}$ by $58 \mathrm{~mm}$ filling defect arising from the inferior and lateral wall of the right atrium. It is worth noting that both peripheral edema and jugular venous distension were absent.

The patient was then referred to the Cardiac Surgery Unit, where he underwent excision of the neoformation by median sternotomy. The patient's postoperative course was uneventful.

Macroscopically, the resected tumor was $5 \mathrm{~cm}$ by $4 \mathrm{~cm}$ by $3 \mathrm{~cm}$, blackish-colored, capsulated, hemorrhagic in appearance, with focal whitish areas infiltrating the myocardium. The pathological examination showed a moderately differentiated cardiac angiosarcoma, with large areas of necrosis and "hemangiopericytoma-like" areas (Fig. 2). The immunohystochemical staining was positive for factor VIII, CD34, vimentin, negative for alfa-actin, bcl-2, panCk.

Twenty-day postoperative assessment with cardiac gadolinium contrast-enhanced MRI, documented a clear $23 \mathrm{~mm}$ by $28 \mathrm{~mm}$ by $24 \mathrm{~mm}$ filling defect with polycyclic profiles and raspberry-like appearance, near the lower portion of the tricuspid annulus. Post-contrast assessment showed a clear heterogeneous enhancement, consistent with residual or recurrent disease. Whole body CT scan with iodinated contrast agent, whole body PET/CT and bone scintigraphy performed for disease staging were negative.

Twenty-four hour urinary catecholamines concentration was normal.

After surgery, the patient showed a significant reduction in blood pressure that allowed progressive tapering of antihypertensive drugs and withdrawal of transdermal clonidine. Furthermore, the epigastric distress disappeared.

The patient was referred to the Oncology Department, where he underwent antiblastic therapy with ifosfamide and doxorubicin.

\section{Discussion}

Dr. Lauro, Dr. Sbraccia: Primary tumors of the heart are rare with an incidence ranging from 0.0017 to $0.0033 \%$ in reported autopsy series. About $75 \%$ of them are benign, the remaining $25 \%$ are malignant, mostly represented by sarcomas. Angiosarcoma, the most frequent malignant histiotype, is a neoplasm of endothelial differentiation more frequently affecting males than females (M:F ratio 2:1), usually between the third and fifth decade of life [2-4]. Angiosarcoma of the heart is characterized by a rapid and
Fig. 1 CT scan (a), transthoracic echocardiography (b)
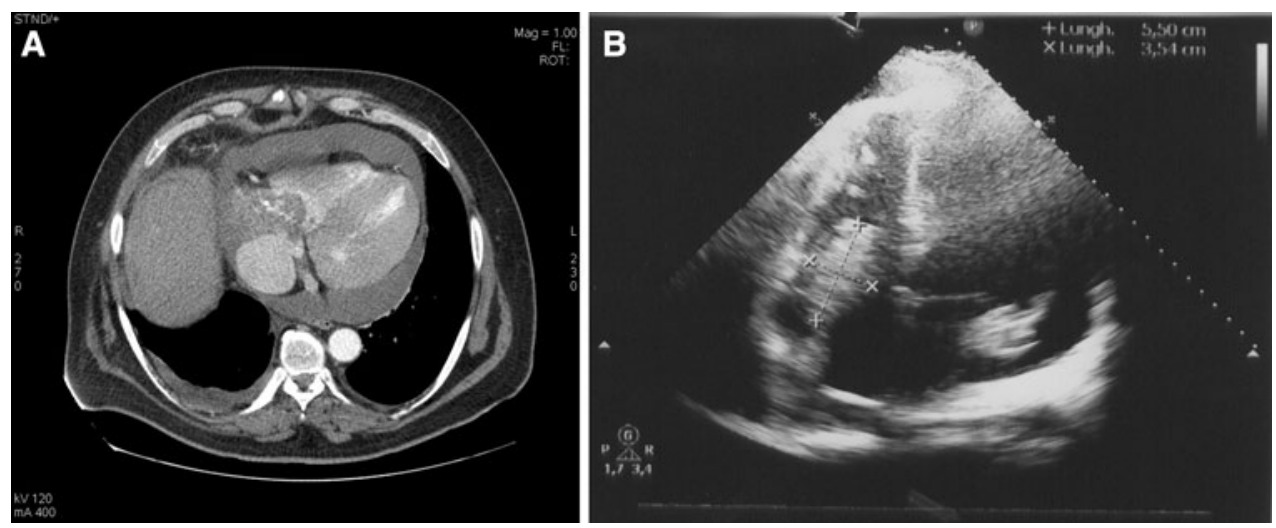


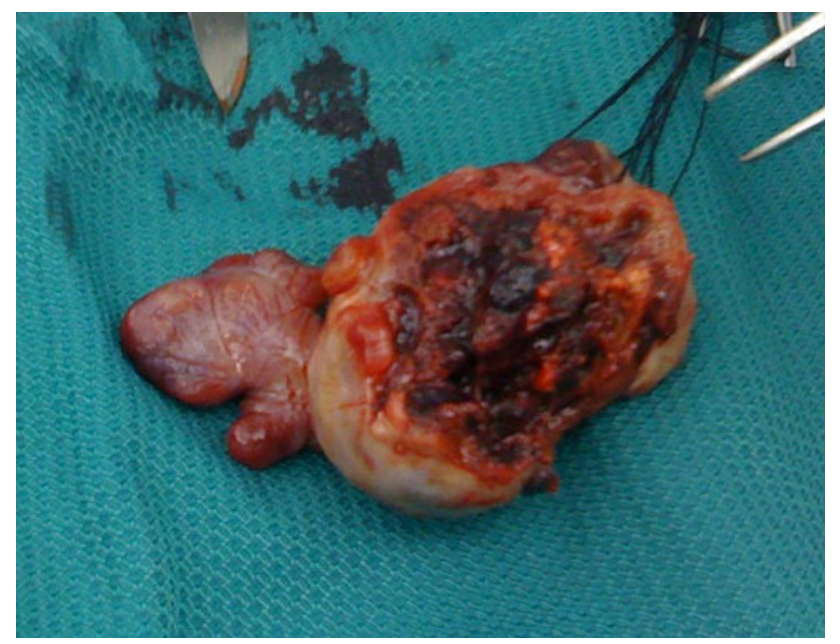

Fig. 2 Photograph of resected tumor

infiltrating growth within the myocardial wall, friability, and tendency toward bleeding, frequently causing pericardial effusion and cardiac tamponade [5]. Another clinical presentation is myocardial rupture due to tumor infiltration and necrosis of the wall [6]. Angiosarcoma is mainly located in the right atrium (78-93\% of cases), pericardium, vena cava and tricuspid valve. At diagnosis, metastases are very common. Presenting symptoms are nonspecific and include dyspnea, chest pain, cough, hemoptysis, syncope, cardiac dysrhythmias, pericardial effusion, tumor embolism, and heart murmurs [7]. Imaging has an important role in early diagnosis and staging. Transthoracic echocardiography especially transesophageal echocardiography can precisely locate the tumor, while computed tomography and MRI have excellent diagnostic advantages with regard to tumor delineation and spread [8]. The main treatment strategy in cardiac angiosarcoma is surgical resection with or without chemotherapy and radiation [9]. However, regardless of treatment, prognosis is poor, with survival ranging from 6 to 12 months [2].

Our patient presented none of the typical symptoms of cardiac angiosarcoma, having only hypertension resistant to treatment along with epigastric pain.

Our patient reported a 10-year history of hypertension, but extreme resistance to treatment only developed before admission. The pathogenesis of hypertension in morbidly obese patients can be explained by several factors, including insulin resistance, alterations in the renin-angiotensinaldosterone system and sympathetic tone [10-14]. Other factors to be considered in linking obesity to hypertension are related to changes in total blood volume, cardiac output, and increase in peripheral vascular resistance, caused either by proinflammatory cytokines, such as IL- 6 and TNF $\alpha$, released from adipose tissue, or by overactivity of the sympathetic nervous system caused by insulin resistance
[15]. Furthermore, more than $50 \%$ of patients with sleep apnea syndrome have overnight high blood pressure levels. This syndrome may cause hypertension because of increased sympathetic tone, chronic proinflammatory state, and endothelial dysfunction [16].

In addition to the above-mentioned factors, the atrial mass might have affected hemodynamics, interfering with the normal cardiac kinetics and most likely inducing a sympathoadrenergic overstimulation, suggestive of a pheochromocytoma. This hypothesis is supported by the postoperative resolution of the treatment-resistant hypertension together with the normalization of the urinary catecholamine concentration.

Conflict of interest None.

\section{References}

1. Chobanian AV, Bakris GL, Black HR et al (2003) Seventh report of the Joint National Committee on prevention, detection, evaluation, and treatment of high blood pressure. Hypertension 42:1206-1252

2. Kontogiorgi M, Exarchos D, Charitos C, Floros I, Rontogianni D, Roussos C, Routsi C (2007) Primary right atrium angiosarcoma mimicking pericarditis. World J Surg Oncol 5:120. doi:10.1186/ 1477-7819-5-120

3. Kurian KC, Weisshaar D, Parekh H, Berry GJ, Reitz B (2006) Primary cardiac angiosarcoma: case report and review of the literature. Cardiovasc Pathol 15:110-112

4. Amonkar GP, Deshpande JR (2006) Cardiac angiosarcoma. Cardiovasc Pathol 15:57-58

5. Antonuzzo L, Rotella V, Mazzoni F, Doni F, Bianchini D, Garbini F, Maio V, Di Costanzo F (2009) Primary cardiac angiosarcoma:a fatal disease. Case Reports Med. doi:10.1155/ 2009/591512

6. Ohri SK, Nihoyannopoulos P, Taylor KM, Keogh BE (1993) Angiosarcoma of the heart causing cardiac rupture: a rare cause of hemopericardium. Ann Thorac Surg 57:259-260

7. Janigan D, Husain A, Robinson N (1986) Cardiac angiosarcomas: a review and a case report. Cancer 57:852-859

8. Koster O, Lackner K, Grube F, Thurn P (1981) Computed tomographic diagnosis of cardiac, pericardial and paracardial space occupying lesions. Z Kardiol 70:733-741

9. Percy RF, Perryman RA, Amornmarn R et al (1987) Prolonged survival in a patient with primary angiosarcoma of the Heart. Am Heart J 113:1228-1230

10. Kolanowski J (1999) Obesity and hypertension: from pathophysiology to treatment. Int J Obes Relat Metab Disord 23:42-46

11. DeFronzo RA (1997) Insulin resistance: a multifaceted syndrome responsible for NIDDM, obesity, hypertension, dyslipidaemia and atherosclerosis. N Engl J Med 50:191-197

12. Ikeda T, Gomi T, Hirawa N, Sakurai J, Yoshikawa N (1996) Improvement of insulin sensitivity contributes to blood pressure reduction after weight loss in hypertensive subjects with obesity. Hypertension 27:1180-1186

13. Tuck ML, Sowers J, Dornfeld L, Kledzik G, Maxwell M (1981) The effect of weight reduction on blood pressure, plasma blood pressure, plasma renin activity, and plasma aldosterone levels in obese patients. N Engl J Med 304:930-933

14. Hsueh WA, Buchanan TA (1994) Obesity and hypertension. Endocrinol Metab Clin North Am 23:405-427 
15. Poirier P, Giles TD, Bray GA, Hong Y, Stern JS, Pi-Sunyer FX, Eckel RH (2006) Obesity and cardiovascular disease: pathophysiology, evaluation and effect of weight loss. Circulation 113:898-918
16. Nieto FJ, Young TB, Lind BK, Shahar E, Samet JM, Redline S, D'Agostino RB, Newman AB, Lebowitz MD, Pickering TG (2000) Association of sleep-disordered breathing, sleep apnea and hypertension in a large community-based study. JAMA 283:1829-1836 